See Article page XXX.

\section{Commentary: Ventricular function improvement after the cone for Ebstein anomaly: It is time to incorporate magnetic resonance studies into every long-term postoperative protocol}

\author{
Emile Bacha, MD, and \\ Rachel D. Vanderlaan, MD, PhD
}

The cone procedure was developed and popularized in the mid-2000s. About 15 to 20 years later, the dominance of this operation over other repairs (eg, Carpentier or Danielson) is firmly established. The operation works and the reconstructed tricuspid valve maintains good function over long periods of time. Ebstein anomaly is a disease of the right ventricular (RV) myocardium, and therefore, the logical question to ask is whether the cone repair affects late biventricular function and ventricular remodeling. Neijenhuis and colleagues ${ }^{1}$ present a single surgeon experience and retrospective analysis of 58 patients who underwent the cone procedure focusing on cardiac magnetic resonance (CMR) outcomes. There was modest improvement in RV function as determined by pulmonary blood flow but, a more dramatic improvement in left ventricular (LV) function. As such, this report is important and supports use of the cone procedure to treat this heterogeneous group of patients.

Patients with Ebstein anomaly have ventricular septal distortion from RV atrialization, dilatation, and dysfunction. In addition, patients can have LV cardiomyopathy, often associated with LV noncompaction. The improvement in

\footnotetext{
From the Division of Pediatric and Congenital Cardiac Surgery, Department of Cardiac, Thoracic, and Vascular Surgery, Columbia University Irving Medical Center, Morgan Stanley Children's Hospital/NewYork-Presbyterian, New York, NY. Disclosures: The authors reported no conflicts of interest.

The Journal policy requires editors and reviewers to disclose conflicts of interest and to decline handling or reviewing manuscripts for which they may have a conflict of interest. The editors and reviewers of this article have no conflicts of interest.

Received for publication Dec 22, 2020; revisions received Dec 22, 2020; accepted for publication Dec 23, 2020.

Address for reprints: Emile Bacha, MD, Division of Pediatric and Congenital Cardiac Surgery, Department of Cardiac, Thoracic, and Vascular Surgery, Columbia University Irving Medical Center, Morgan Stanley Children's Hospital/NewYorkPresbyterian, New York, NY 10032 (E-mail: eb2709@cumc.columbia.edu).

J Thorac Cardiovasc Surg 2021; $\mathbf{0}: 1-2$ $0022-5223 / \$ 36.00$

Copyright (c) 2020 Published by Elsevier Inc. on behalf of The American Association for Thoracic Surgery

https://doi.org/10.1016/j.jtcvs.2020.12.106
}

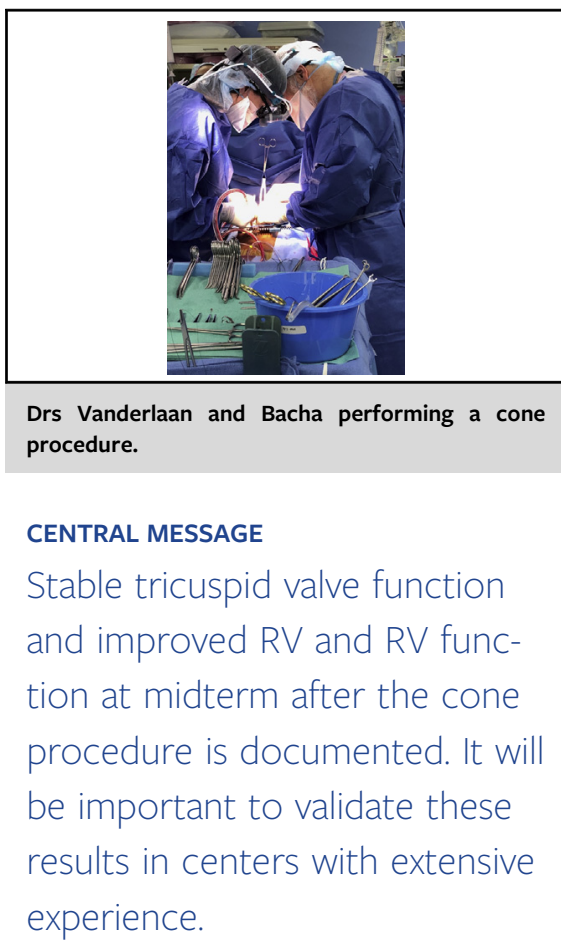

LV function after cone reconstruction could be related to 2 main phenomena: a ventricular-ventricular interactiontype phenomenon related to RV geometrics and improved synchrony, or it could be related to improvement in LV filling (ie, preload) from improved overall RV functional efficiency. The present CMR analysis did not specifically assess septal motion. Therefore, as with many good and worthwhile studies, this report reveals more questions than it answers. The reason for the recovery of LV function (which other authors have noted ${ }^{2,3}$ ), if true, is unknown as of yet.

It is also important to note that these results are based on a small number of patients $(n=12)$ who actually had CMR examinations before and after. Therefore, the next order of business for the congenital heart surgery community is to validate these results. Every patient who has had a cone reconstruction should have a scheduled CMR at mid- to long-term postoperative follow-up. Only then, with a larger number of patients studied, will we know whether the cone operation, beyond providing a durable repair at the valve level, also results in improvement in RV and LV function and why.

\section{References}

1. Neijenhuis RML, Tsang VT, Marek J, Issitt R, Bonello B, Van Klemperer K, et al Cone reconstruction for Ebstein anomaly: late biventricular function and possible remodeling. J Thorac Cardiovasc Surg. 2021 [In press]. 
2. Beroukhim RS, Jing L, Harrild DM, Fornwalt BK, Mejia-Spiegeler A, Rhodes J, et al. Impact of the cone operation on left ventricular size, function, and dyssynchrony in Ebstein anomaly: a cardiovascular magnetic resonance study. Cardioyasc Magn Reson. 2018;20:32.
3. Stähli BE, Gebhard C, Biaggi P, Klaassen S, Valsangiacomo Buechel E, Attenhofer Jost CH, et al. Left ventricular non-compaction: prevalence in congenital heart disease. Int J Cardiol. 2013;167:2477-81. 
000 Commentary: Ventricular function improvement after the cone for Ebstein anomaly: It is time to incorporate magnetic resonance studies into every longterm postoperative protocol

Emile Bacha, MD, and Rachel D. Vanderlaan, MD, PhD, New York, NY

Stable tricuspid valve function and improved RV and RV function at midterm after the cone procedure is documented. It will be important to validate these results in centers with extensive experience. 